\title{
Fluorescence Photoswitching in a Series of Metal-Organic Frameworks Loaded with Different Anthracenes
}

\author{
Min Tu, ${ }^{[a, b]}$ Mariana Lizeth Díaz Ramírez, ${ }_{1}^{[c]}$ llich A. Ibarra, ${ }^{[c]}$ Johan Hofkens, ${ }^{[d, e]}$ and \\ Rob Ameloot*[a]
}

The confinement of luminescent guest molecules in porous host materials can induce photophysical properties different from either component in isolation. In this work, we studied several host-guest systems consisting of anthracene and its substituted analogs adsorbed in a series of metal-organic

\section{Introduction}

Photoswitchable materials undergo reversible changes in their physicochemical properties (e.g., absorbance, fluorescence, electron conductivity) by light irradiation. ${ }^{[1-3]}$ These materials typically contain molecules that switch between two stable forms, such as trans- and cis-azobenzene, ${ }^{[4]}$ the open and closed form of diarylethenes, ${ }^{[5]}$ or anthracene monomer and photodimer. ${ }^{[6,7]}$ Fluorescence photoswitching of the emission intensity or color in solid-state materials is promising for data storage, anti-counterfeiting, security, and biomedicine. ${ }^{[8-11]}$ Unfortunately, many of the switching characteristics in the solution are not readily transferable to the solid state since the switching mechanism is hindered in a densely packed phase. One promising strategy to overcome this challenge is to confine photoswitchable guests in porous solids, such as zeolites, mesoporous silica, and metal-organic frameworks (MOFs). ${ }^{[12-22]}$

Crystalline MOFs are nanoporous solids that consist of metal ion nodes connected by multi-topic organic molecules. ${ }^{[23,24]}$ The properties of MOFs, including structural flexibility and tunable pore size, are ideal to confine photoswitchable guests in a

[a] Dr. M. Tu, Prof. R. Ameloot

Centre for Membrane Separations, Adsorption, Catalysis, and Spectroscopy (cMACS)

KU Leuven - University of Leuven

Celestijnenlaan 200F, 3001 Leuven, Belgium

E-mail: rob.ameloot@kuleuven.be

[b] Dr. M. Tu

2020 X-Lab, Shanghai Institute of Microsystem and Information Technology, Chinese Academy of Science Shanghai, Shanghai, 200050, China

[c] M. L. Díaz Ramírez, Prof. I. A. Ibarra

Laboratorio de Fisicoquímica y Reactividad de Superficies (LaFReS), Instituto de Investigaciones en Materiales,

Universidad Nacional Autónoma de México,

Ciudad de México, Mexico

[d] Prof. J. Hofkens

Department of Molecular Visualization and Photonics,

KU Leuven-University of Leuven,

Celestijnenlaan 200F, 3001 Leuven, Belgium

[e] Prof. J. Hofkens

Max Planck Institute for Polymer Research,

Ackermannweg 10, 55128 Mainz, Germany

Supporting information for this article is available on the WWW under https://doi.org/10.1002/ejic.202100415 frameworks (MOFs) and inorganic molecular sieves. Fluorescence photoswitching of the guest molecules through photoinduced dimerization is observed only in MOFs with a favorable pore volume and geometry.

variety of nanoenvironments. ${ }^{[14-16]}$ We recently reported reversible solid-state photoswitching of fluorescence through the confinement of anthracene (ANT) in a MOF (zeolitic imidazolate framework 8, ZIF-8). ${ }^{[25]}$ We demonstrated that four ANT molecules organize as two pairs in the cages of ZIF-8, giving rise to excimer emission (maximum at $550 \mathrm{~nm}$, yellow). Upon irradiation of UV light, the limited space in the cage only permits two ANT molecules to photodimerize, resulting in one non-fluorescent ANT photodimer and two non-interacting ANT molecules that emit as monomers (maximum at $415 \mathrm{~nm}$, purple). This phenomenon relies on the match between the ANT guest and the properties of ZIF-8, namely its pore geometry, pore volume, and structural flexibility. ${ }^{[25]}$

In this work, we encapsulated ANT in several MOFs and inorganic molecular sieves (Figure 1). The differences in pore size, volume, and structural flexibility of the porous host materials resulted in diverse fluorescence properties of these host-guest systems. Moreover, we demonstrated that substituted anthracenes (2-methylanthracene, 2-MA; 9-methylanthracene, 9-MA) in ZIF-8 show similar fluorescence photoswitching behavior as ANT-loaded ZIF-8.

\section{Results and Discussion}

The MOFs MAF-6 (metal azolate framework 6) and ZIF-71 consist of $\mathrm{Zn}^{2+}$ coordinated by 2-ethylimidazolate (elm) and 4,5-dichloroimidazolate (dclm), respectively (Figure 1). ${ }^{[26,27]}$ Both materials crystallize in the zeolitic RHO topology, resulting in truncated cuboctahedral supercages interconnected by octagonal prisms and an effective pore diameter and aperture size of 1.8 and $0.7 \mathrm{~nm}$, respectively. ${ }^{[26,28]}$ For comparison, ZIF-8 has spherical cavities of $1.16 \mathrm{~nm}$ in diameter, interconnected by narrow $0.34 \mathrm{~nm}$ windows. ${ }^{[25]}$ Although these windows seem too small to permit ANT adsorption, a high loading can be achieved thanks to the framework flexibility. ${ }^{[25]}$ Similarly, ANT can be readily loaded into MAF-6 and ZIF-71 via sublimation. The ANT uptake was determined by thermogravimetric analysis (TGA; Figure 2a). Due to their large pore volume, the maximum ANT loading in MAF-6 and ZIF-71 were 7.2 and 7.4 molecules per 
(a)

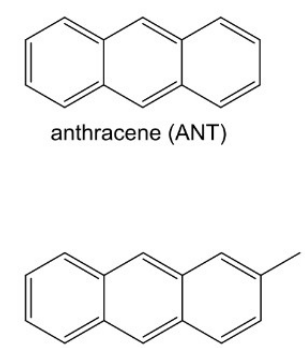

2-methylanthracene (2-MA)

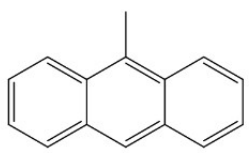

9-methylanthracene (9-MA) (b)

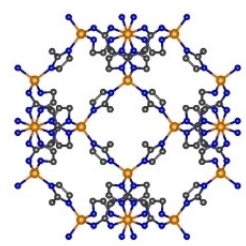

ZIF-8
Fluorescence photoswitching

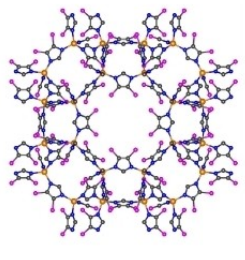

ZIF-71

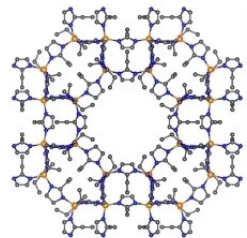

MAF-6

No fluorescence photoswitching

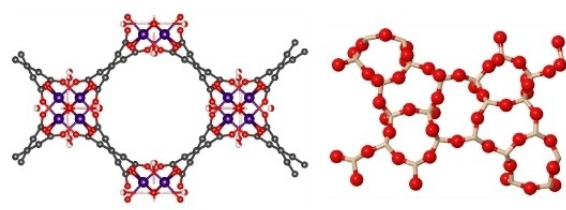

MFM-300 (Sc)

Silicalite-1

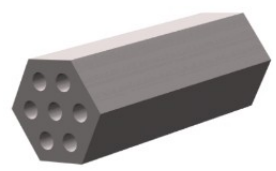

SBA-15

Figure 1. Anthracene guests and porous hosts. (a) Chemical structures of ANT, 2-MA, and 9-MA. (b) Ball-stick representation of crystal structures of ZIF-8, ZIF71, MAF-6, MFM-300 (Sc) and Silicalite-1. All views from b axis. Schematic representation of mesoporous SBA-15. Zn, orange; N, blue; C, grey; Cl, pink; O, red; Sc, purple; Si, brown.

(a)

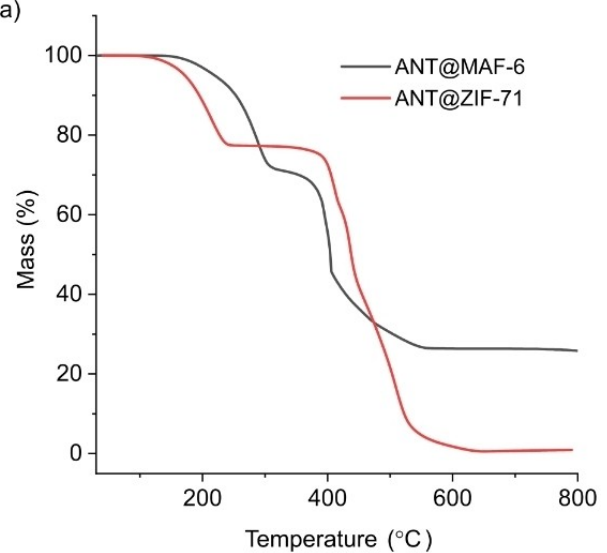

(b)
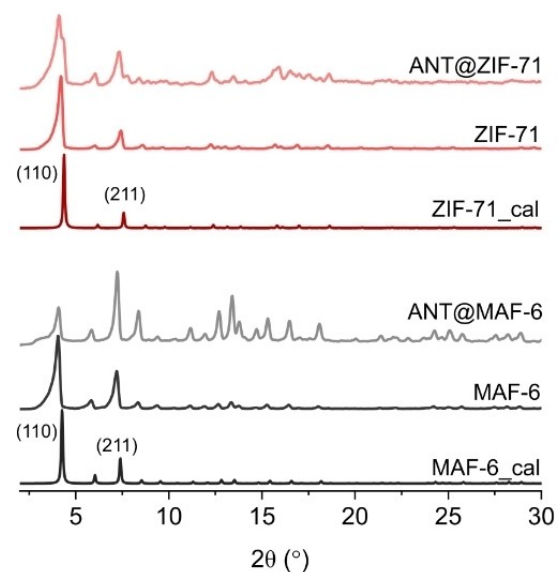

Figure 2. Characterization of ANT-loaded MAF-6 and ZIF-71. (a) TGA of ANTloaded MAF-6 and ZIF-71 in air. The ANT loadings for MAF-6 and ZIF-71 were determined to be $30 w t \%$ and $24 w t \%$, respectively. (b) PXRD patterns (normalized intensity) of MAF-6 and ZIF-71 before and after ANT loading. The calculated PXRD patterns of the corresponding guest-free MOFs are given for reference. cage, respectively. These loadings are almost double the maximum loading in ZIF-8 (4 ANT per cage). ${ }^{[25]}$ Powder X-ray diffraction (PXRD) showed that the long-range order is maintained in both MOFs after ANT loading (Figure 2b). The splits of the low-angle reflections of ANT@ZIF-71 indicated a distortion of the unit cell, similar to ANT@ZIF-8. ${ }^{[25]}$ The encapsulation of ANT in MAF-6 and ZIF-71 also resulted in UV-induced fluorescence switching. The emission spectra of ANT@MAF-6 and ANT@ZIF-71 showed broad bands centered at 550 and $500 \mathrm{~nm}$, respectively (Figure 3a, Figure 3c). These emissions are attributed to ANT excimers, whose emission is highly dependent on the arrangement of the two ANT molecules. ${ }^{[29,30]}$ The slightly different pore environments of MAF- 6 and ZIF-71 likely result in different ANT organizations and excimer fluorescence. After continuous UV irradiation $(\lambda=360 \mathrm{~nm})$, several structured bands appeared for both ANT-loaded MOFs, together with the disappearance of the band at 550 or $500 \mathrm{~nm}$ (Figure 3a, Figure $3 \mathrm{c}$ ), due to ANT photodimerization and remaining monomer emission. In situ fluorescence monitoring also revealed the enhancement of the monomer emission (Figure $3 b$, Figure $3 d$ ).

The effect of loading on the fluorescence photoswitching was studied in the ANT@ZIF-71 host-guest system. Different ANT loadings were obtained by varying the ANT:ZIF-71 ratio during sublimation. PXRD patterns of ANT@ZIF-71 showed all the characteristic reflections of ZIF-71 (Figure 4a). With increasing ANT loading, the Brunauer-Emmett-Teller (BET) surface area of the host-guest composites decreased (Figure 4b). At low loadings ( $<1.5$ ANT per cage), monomer emission dominated the emission spectra with only a small broad band centered at $550 \mathrm{~nm}$ (Figure 4c). In contrast, broad asymmetric bands appeared for higher loadings, probably because of two different organizations of the ANT pairs and resulting excimer emission bands centered at $430 \mathrm{~nm}$ and $550 \mathrm{~nm} \cdot{ }^{[31]}$ Furthermore, the ANT 
(a)

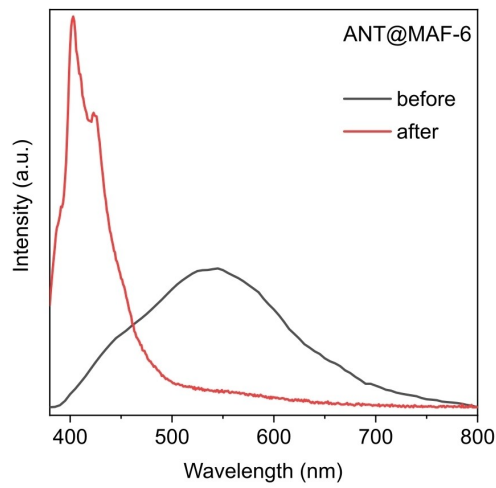

(c)

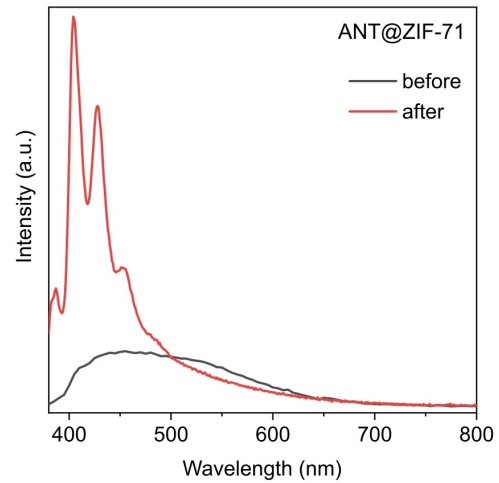

(b)
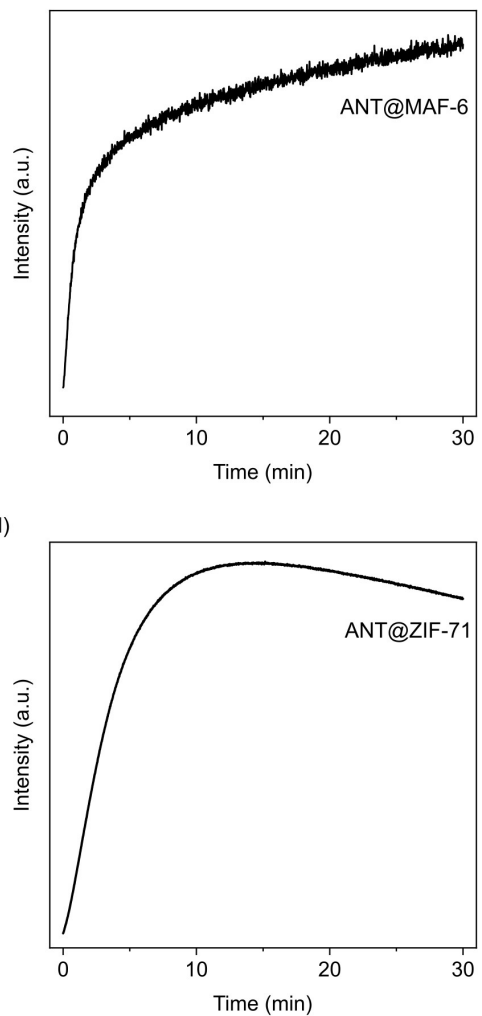

Figure 3. Fluorescence photoswitching of ANT-loaded MOFs. Fluorescence emission spectra of ANT@MAF-6 (a) and ANT@ZIF-71 (c) before and after UV irradiation ( $\lambda=360 \mathrm{~nm} ; 30 \mathrm{~min}) . \lambda_{\text {ex }}=360 \mathrm{~nm}$. In situ monitoring of the fluorescence emission intensity of ANT@MAF-6 (b; $\left.\lambda_{\text {em }}=415 \mathrm{~nm}\right)$ and ANT@ZIF-71 (d; $\lambda_{\mathrm{em}}=400 \mathrm{~nm}$ ) upon continuous irradiation at $\lambda=360 \mathrm{~nm}$.

(a)

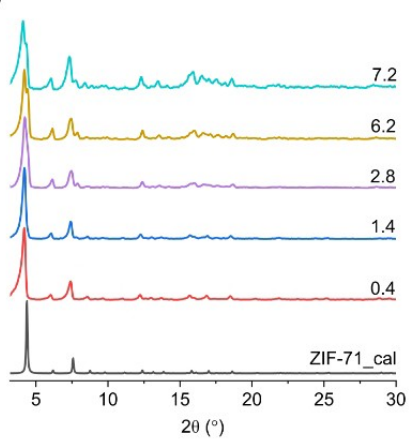

(c)

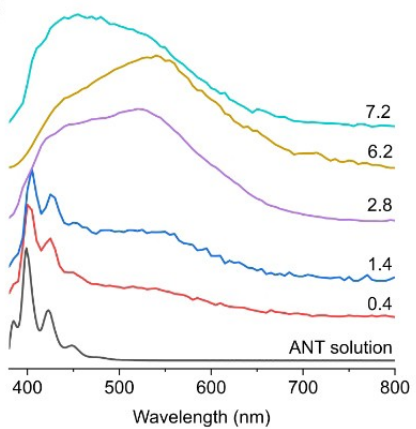

(b)

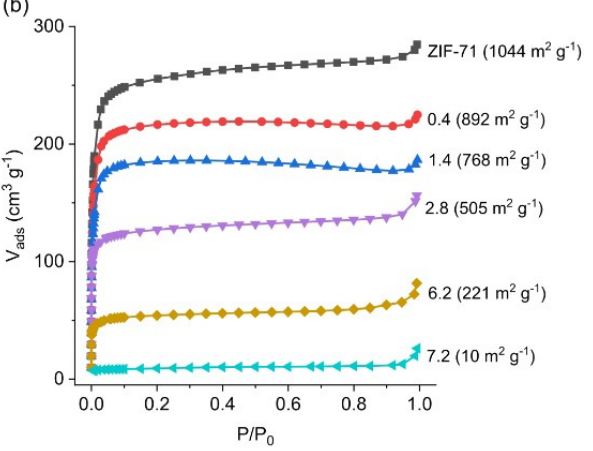

(d)

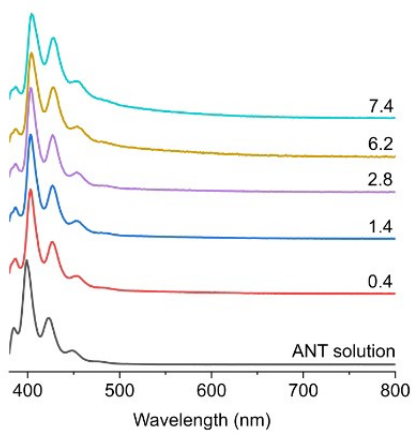

Figure 4. Characterization and photoswitching of ANT@ZIF-71 with different guest loadings. (a) PXRD patterns (normalized intensity) of ZIF-71 with different loadings (ANT per cage). The calculated PXRD pattern of ZIF-71 is given for reference. (b) $\mathrm{N}_{2}$ physisorption isotherms (at 77 K) of ANT@ZIF-71 with different loadings and the corresponding BET surface areas. Fluorescence emission spectra (normalized intensity) of ANT@ZIF-71 with different loadings before (c) and after (d) UV irradiation ( $\lambda=360 \mathrm{~nm} ; 30 \mathrm{~min}) . \lambda_{\text {ex }}=360 \mathrm{~nm}$. 
monomer emission after UV irradiation is red-shifted for highloading ANT@ZIF-8 (Figure S6). ${ }^{[25]}$ This phenomenon was attributed to the intermolecular interaction between ANT molecules in neighboring cages. However, UV-irradiated ANT@ZIF-71 exhibited the same fluorescence band $\left(\lambda_{\max }=405 \mathrm{~nm}\right.$; Figure $4 d$ ) irrespective of the ANT loading, probably because the prisms separating the cages prohibited interactions between ANT molecules in neighboring cages.

MFM-300(Sc) crystallizes in the chiral tetragonal space group and shows a binuclear $\left[\mathrm{Sc}_{2}(\mu-\mathrm{OH})\right]$ node, leading to a three-dimensional framework with a pore opening of $0.8 \mathrm{~nm}$ (Figure 1b). ${ }^{[28]}$ After the sublimation of ANT into MFM-300(Sc), the relative intensity changes of the PXRD reflections confirm the loading in the pores (Figure 5a), and the TGA data suggested a loading of 1.8 ANT per pore (Figure S7). Similar to
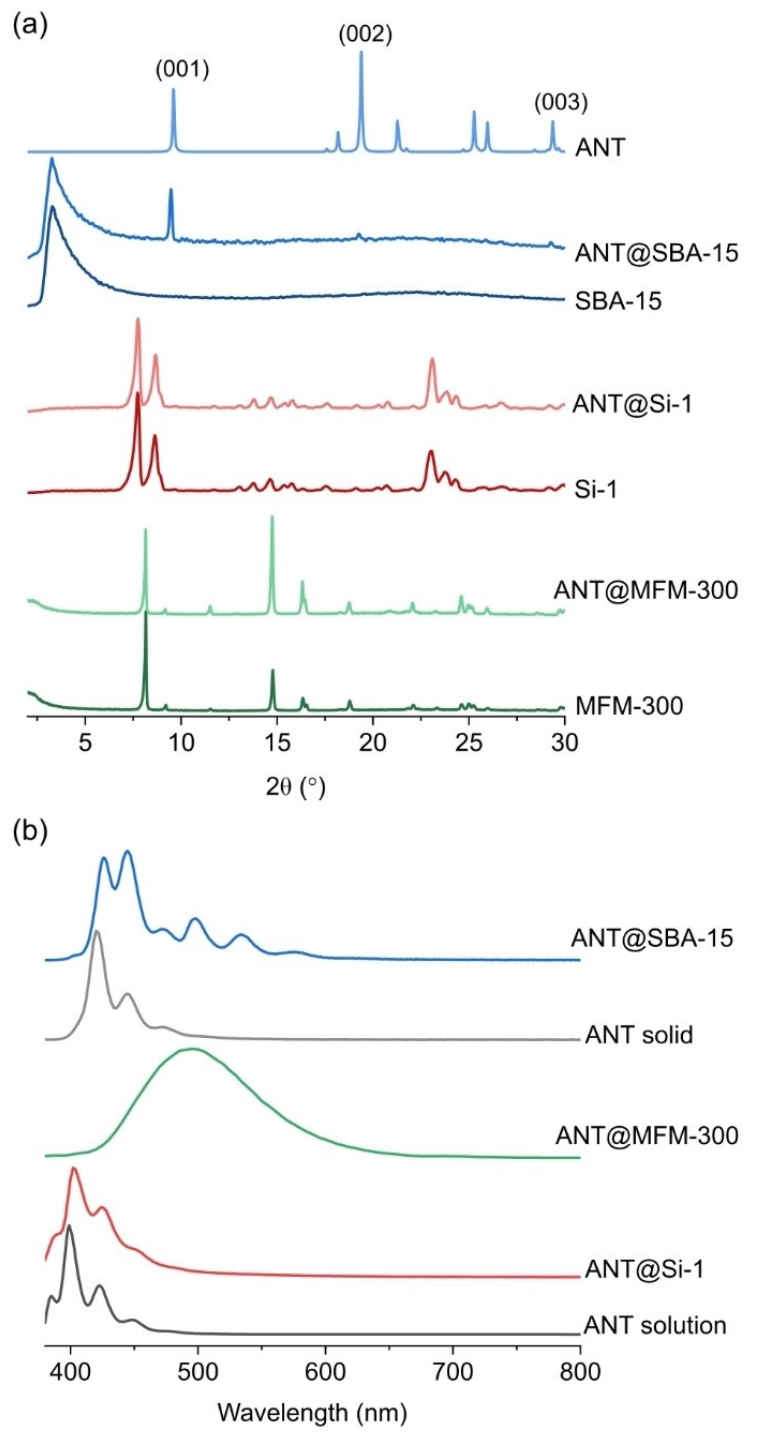

Figure 5. Characterization of ANT-loaded MFM-300(Sc), Si-1 and SBA-15. (a) PXRD patterns (normalized intensity) of MFM-300(Sc), Si-1 and SBA-15 with and without ANT loading. PXRD pattern of ANT solid is given for reference. (b) Fluorescence emission spectra of ANT@MFM-300(Sc), ANT@Si-1 and ANT@SBA-15 in comparison with ANT methanol solution (1 mM) and solid. $\lambda_{\text {ex }}=360 \mathrm{~nm}$.
ANT in MAF-6 and ZIF-71, the fluorescence exhibited a broad excimer emission band $\left(\lambda_{\max }=500 \mathrm{~nm}\right.$; Figure $5 \mathrm{~b}$ ). The two ANT molecules per pore can form an organized pair, giving rise to excimer emission. However, no photoswitching from excimer to monomer emission was observed after UV irradiation (Figure S8).

Similar to MOFs, inorganic molecular sieves such as zeolites and mesoporous silicas also have uniform pore sizes. We studied ANT-loaded molecular sieves with different pore diameters, including Silicalite-1 (Si-1) and SBA-15. Si-1 is a puresilica zeolite with an MFI topology (Figure 1b). It has straight channels $0.51 \times 0.55 \mathrm{~nm}^{2}$ intersecting perpendicularly with sinusoidal channels $0.52 \times 0.56 \mathrm{~nm}^{2}$. $^{[33]}$ Due to this narrow channel diameter, the uptake of ANT was hindered, resulting in a low loading by sublimation ( 2 wt\%; Figure S7) and a remaining BET surface area of $277 \mathrm{~m}^{2} \mathrm{~g}^{-1}$ (Figure S9). In contrast, full loading could be achieved in ZIF-8, regardless of its smaller theoretical pore windows, due to the high flexibility of the MOF material. Because of its small pore size and strict confinement, ANT@Si-1 exhibited monomer emission, and no photoswitching was observed (Figure 5b, S8), which is in agreement with results for solution-loaded ANT in ZSM-5 ( $\mathrm{Na}^{+}$form). ${ }^{[33]}$

SBA-15 has uniform one-dimensional hexagonal mesopores that can be controlled in the range from 4 to $30 \mathrm{~nm} \cdot{ }^{[34]}$ Here, 6nm-pore SBA-15 was employed, which allows high ANT loadings (20 wt\%; Figure S7). After ANT loading, several new reflections matching crystalline ANT appeared in the PXRD pattern (Figure $5 \mathrm{a}){ }^{[35]}$ suggesting the crystallization of the guest in the mesopores. ${ }^{[36]}$ The fluorescence spectrum of ANT@SBA-15 was very similar to what would be expected for solid ANT (Figure 5b). In the crystalline state, ANT forms a herringbone arrangement with small $\pi$-orbital overlap owing to strong edge-to-face $\mathrm{CH}-\pi$ interactions (Figure S14). ${ }^{[35]}$ Therefore, the ANT solid exhibits the monomer emission, which is slightly redshifted compared to the monomer emission of a solution in methanol (Figure 5b). ${ }^{[37]}$ Similar to solid ANT, no photoswitching was observed for ANT-loaded SBA-15 (Figure S12).

The substituted ANTs 2-MA and 9-MA can also photodimerize upon UV irradiation. ${ }^{[6,7]}$ However, because of their larger size compared to ANT, they could not be loaded effectively into ZIF-8 (Figure S15-16). Therefore, a 'bottlearound-the-ship' encapsulation strategy was used, by having the guest molecule present during the solvent-free crystallization of ZIF-8 (details see Experimental Section). ${ }^{[38]}$ The loadings (0.1-3 molecules per cage) could be controlled through the 2MA or 9-MA content in the synthesis mixture (Figure S17-18). The PXRD patterns demonstrated the formation of ZIF-8 (Figure $6 a$, S18) with different loadings. Similar to ANT loading in ZIF-8, the increase of loading gave rise to more intense (200) and (211) reflections. ${ }^{[25]}$

Monomer emission was observed for low loadings, while excimer emission dominated at high loadings (Figure 6b). Similar to ANT in ZIF-8, UV-induced fluorescence switching of high-loading 9-MA@ZIF-8 from excimer emission to monomer emission was observed (Figure 6b-f). Different from ANT@ZIF-8, the excimer emission diminished but still existed after UV irradiation (Figure 6d). Furthermore, likely due to the intermo- 
(a)

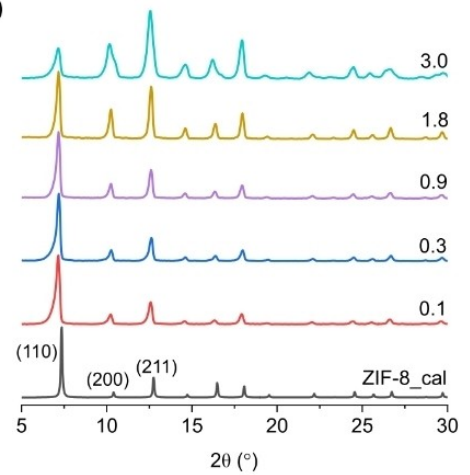

(d)

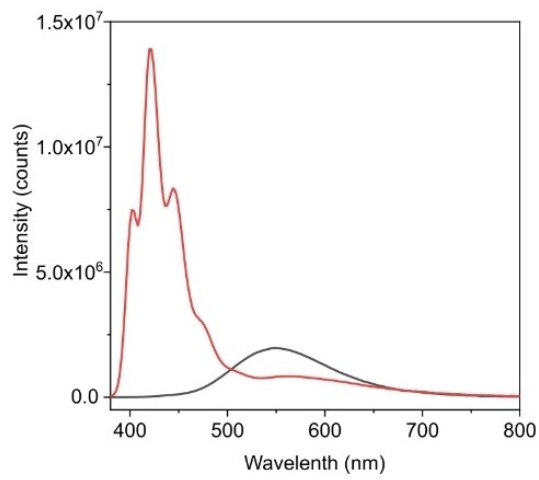

(b)

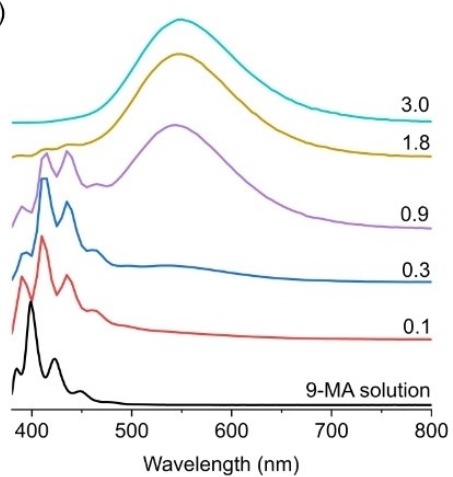

(e)

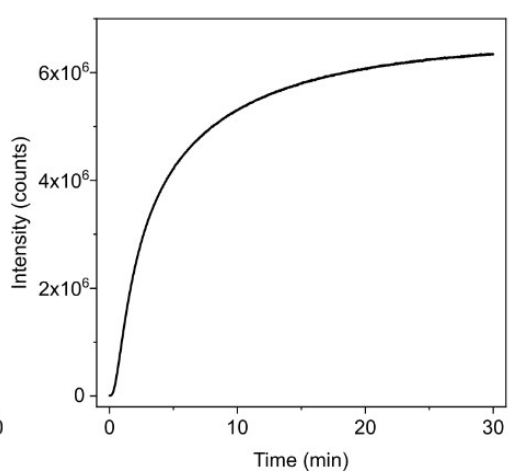

(c)

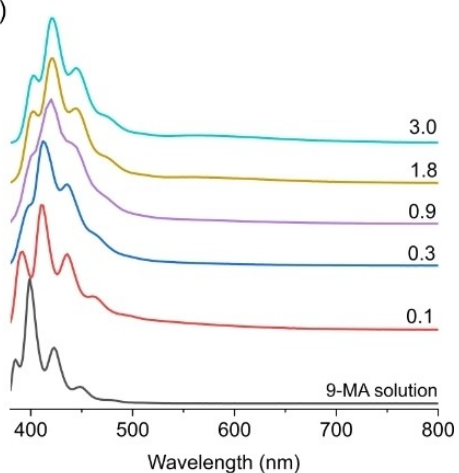

(f)

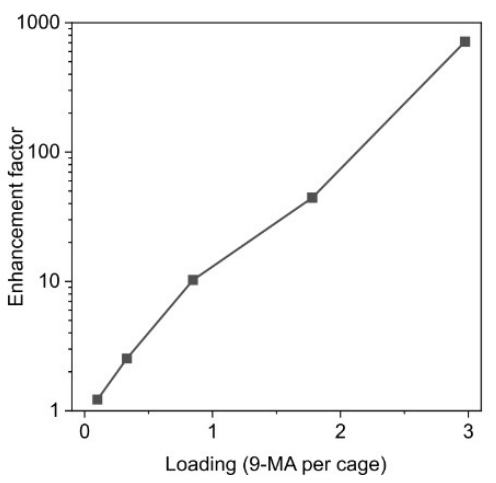

Figure 6. Characterization and photoswitching of 9-MA@ZIF-8. (a) PXRD patterns (normalized intensity) of ZIF-8 with different loadings (9-MA per cage). The calculated PXRD pattern of ZIF-8 is given for reference. Fluorescence emission spectra (normalized intensity) of 9-MA@ZIF-8 with different loadings before (b) and after (c) UV irradiation ( $\lambda=360 \mathrm{~nm} ; 30 \mathrm{~min}$ ). $\lambda_{\mathrm{ex}}=360 \mathrm{~nm}$. (d) Fluorescence emission spectra (absolute intensity) of 9-MA@ZIF-8 (3.0) before and after UV irradiation ( $\lambda=360 \mathrm{~nm} ; 30 \mathrm{~min}$ ). $\lambda_{\mathrm{ex}}=360 \mathrm{~nm}$. (e) In situ monitoring of the fluorescence emission intensity of 9-MA@ZIF-8 ( $\lambda_{\mathrm{em}}=415 \mathrm{~nm}$ ) upon continuous excitation at $\lambda=360 \mathrm{~nm}$. (f) Fluorescence enhancement of 9-MA@ZIF-8 as a function of the 9-MA loading. The enhancement factor is defined as the ratio of monomer emission intensity $(\lambda=415 \mathrm{~nm})$ after and before UV irradiation.

lecular interactions of 9-MA in the neighboring cages, the monomer emission of UV-irradiated 9-MA@ZIF-8 sample was red-shifted at high 9-MA loadings. 2-MA@ZIF-8 also showed similar photoswitching behavior as 9-MA loaded ZIF-8 (Figure S24-26).

\section{Conclusion}

In summary, we studied the photophysical behavior of anthracene and its substituted analogs in different porous materials. Similar to ANT@ZIF-8, fluorescence photoswitching was observed for ANT-loaded MAF-6 and ZIF-71, and 2-MA and 9-MA-loaded ZIF-8. In contrast, no photoswitching was observed for ANT in MFM-300(Sc), Si-1, and SBA-15, due to unsuitable pore sizes and geometries. This work will aid further studies on solid-state photoswitching of host-guest systems based on microporous crystalline materials such as MOFs as hosts.

\section{Experimental Section}

\section{Materials}

All chemicals were purchased from commercial supplies and used without further purification. The abbreviations, supplies, and purities of the chemicals are given below. Pluronic P123 (SigmaAldrich), tetraethyl orthosilicate (TEOS, Acros, 98\%), zinc oxide ( $\mathrm{ZnO}, 25 \mathrm{~nm}$, Carl Roth, 99\%), $\mathrm{Zn}\left(\mathrm{CH}_{3} \mathrm{COO}\right)_{2} \cdot 2 \mathrm{H}_{2} \mathrm{O}\left(\mathrm{Zn}(\mathrm{OAc})_{2} \cdot 2 \mathrm{H}_{2} \mathrm{O}\right.$, Sigma-Aldrich, 98\%), 2-methylimidazole (Hmlm, Sigma-Aldrich, 99\%), 2-ethylimidazole (Helm, Sigma-Aldrich, 98\%), 4, 5-dichloroimidazole (Hdclm, TCl, 97\%), anthracene (ANT, Sigma-Aldrich, 98\%), 2-methylanthracene (2-MA, Sigma-Aldrich, 97\%), 9-methylanthracene (9-MA, Sigma-Aldrich, 98\%), biphenyl-3,3',5,5'-tetracarboxylic acid $\left(\mathrm{H}_{4} \mathrm{BTPC}\right.$, Sigma-Aldrich), scandium triflate $\left(\mathrm{Sc}\left(\mathrm{SO}_{3} \mathrm{CF}_{3}\right)_{3}\right.$, Sigma-Aldrich, 99\%), concentrated $\mathrm{HCl}$ (37 wt.\%, Sigma-Aldrich), tetrahydrofuran (THF, Sigma-Aldrich, 99\%), dimethylformamide (DMF, Sigma-Aldrich, 98\%), dichloromethane (DCM, Sigma-Aldrich, $99 \%)$, and methanol (MeOH, Sigma-Aldrich, 99\%) and Si-1 (Tosoh).

\section{Synthesis}

MFM-300 (Sc): ${ }^{[32]} 10 \mathrm{mg} \quad \mathrm{H}_{4} \mathrm{BTPC}$ and $30 \mathrm{mg} \mathrm{Sc}\left(\mathrm{SO}_{3} \mathrm{CF}_{3}\right)_{3}$ were dissolved in a solvent mixture of THF $(4.0 \mathrm{~mL}))$, DMF $(3.0 \mathrm{~mL})$, deionized $\mathrm{H}_{2} \mathrm{O}(1.0 \mathrm{~mL})$ and concentrated $\mathrm{HCl}(100 \mu \mathrm{L})$. The mixture was stirred until complete dissolution $\mathrm{o}$ and then transferred to a pressure tube. The tube was placed in an oil bath and heated to $348 \mathrm{~K}$ for $72 \mathrm{~h}$. After cooling down to room temperature, the 
crystalline product was recovered by filtration, washed with DMF $(5 \mathrm{~mL})$, acetone-exchanged, and dried in air.

MAF-6: ${ }^{[39]}$ A physical mixture of ZnO (162 mg) and 2-ethylimidazole (Helm, $576 \mathrm{mg}$ ) was prepared in glass synthesis bottles. The capped bottle was placed in a preheated oven at a temperature of $80^{\circ} \mathrm{C}$ for 1 day.

ZIF-71: $:^{[40]} 74 \mathrm{mg} \mathrm{Zn}(\mathrm{OAc})_{2} \cdot 2 \mathrm{H}_{2} \mathrm{O}$ was dissolved in $15 \mathrm{~mL}$ methanol, and $220 \mathrm{mg} \mathrm{Hdclm}(220 \mathrm{mg}$ ) was dissolved in $15 \mathrm{~mL}$ methanol. After complete dissolution, the $\mathrm{Zn}(\mathrm{OAc})_{2} \cdot 2 \mathrm{H}_{2} \mathrm{O}$ solution was poured $\mathrm{Hdclm}$ solution. The mixture left standing at room temperature for 1 day.

ZIF-8: ${ }^{[41]} 68 \mathrm{mg} \mathrm{Zn}\left(\mathrm{NO}_{3}\right)_{2} \cdot 6 \mathrm{H}_{2} \mathrm{O}$ was dissolved in $5 \mathrm{~mL}$ methanol, and $41 \mathrm{mg} \mathrm{Hmlm}$ was dissolved in $5 \mathrm{~mL}$ methanol. After complete dissolution, the $\mathrm{Zn}\left(\mathrm{NO}_{3}\right)_{2} \cdot 6 \mathrm{H}_{2} \mathrm{O}$ in methanol solution was poured into the $\mathrm{mlm}$ in methanol solution. The mixture was left standing at room temperature for 1 day.

All the as-synthesized MOF powder samples were collected by centrifugation and washed with methanol by three repetitions of sonication-centrifugation to remove unreacted chemicals. After washing, the ZIF powders were immersed into DCM for 1 day, and subsequently activated at $120^{\circ} \mathrm{C}$ under reduced pressure $(0.5 \mathrm{mbar})$ for 6 hours.

SBA-15: ${ }^{[42]}$ Pluronic P123 (20 g) was dissolved in deionized $\mathrm{H}_{2} \mathrm{O}$ $(635 \mathrm{~g})$ and concentrated $\mathrm{HCl}$ at $35^{\circ} \mathrm{C}$. After a full $\mathrm{P} 123$ dissolution, TEOS (43 g) was added for the synthesis of $12 \mathrm{~g}$ of silica. This mixture was stirred for $20 \mathrm{~h}$ at $35^{\circ} \mathrm{C}$ and afterwards filtered and washed. The recovered powder was dried overnight at $80^{\circ} \mathrm{C}$ and finally calcined at $550^{\circ} \mathrm{C}$ for $6 \mathrm{~h}$ (ramp rate: $1^{\circ} \mathrm{C} \mathrm{min}^{-1}$ ).

ANT loading: To achieve high loading, vials containing a porous material $(100 \mathrm{mg})$ and an excess amount of ANT $(300 \mathrm{mg})$ were placed together in a Schlenk tube. After the evacuation, the Schlenk tube was placed in a preheated oven $\left(120^{\circ} \mathrm{C}\right)$ under a static vacuum $\left(10^{-1} \mathrm{mbar}\right)$ for 3 days. Afterwards, the samples were collected for different characterizations without further treatment. Different ANT loadings in ZIF-71 $(0.4,1.4,2.8,6.2$, and 7.2 molecules per cage) were obtained by varying the amount of ANT $(5,10,25,50$, and $100 \mathrm{mg}$, respectively).

2-MA and 9-MA loading in ZIF-8: The synthesis of 9-MA@ZIF-8 was performed as follows. A mixture of $\mathrm{ZnO}$ (162 mg), Hmlm (492 mg), and various amounts of 9-MA $(10,30,60,100$, and $200 \mathrm{mg})$ was ground in an agate mortar and then transferred into a $10 \mathrm{~mL}$ glass vial. Subsequently, the vial was placed into a preheated convection oven at $110^{\circ} \mathrm{C}$ for 1 day. Afterwards, the obtained powder sample was washed with methanol ( $\times 3$ times) to remove the excess $\mathrm{Hmlm}$ and 9-MA. Finally, the samples were dried at $60^{\circ} \mathrm{C}$ in air. The resulted loadings (9-MA per cage) based on this recipe are $0.18,0.7$, $1.42,2.62$, and 3.21. The encapsulation of 2-MA in ZIF-8 was synthesized in a similar fashion.

\section{Methods}

PXRD data were recorded on an STOE COMBI P diffractometer with CuK $\alpha 1$ radiation $(\lambda=1.54060 \AA)$ and an IP-PSD detector in transmission geometry. Solution ${ }^{1} \mathrm{H}$ NMR spectra were recorded on a Bruker Advance $300 \mathrm{MHz}$ spectrometer. The dried ANT, 2-MA and 9MA-loaded MOF powder (ca. $2 \mathrm{mg}$ ) samples were dissolved in a solution containing $\mathrm{DCl} / \mathrm{D}_{2} \mathrm{O}(32 \mathrm{wt} \%, 50 \mu \mathrm{L})$ and $500 \mu \mathrm{L}$ DMSO- $\mathrm{d}_{6}$. TGA was carried out on a Netzsch STA 449F3 instrument with a heating rate of $5^{\circ} \mathrm{Cmin}^{-1}$ under air. The ANT loading (molecule per cage, $x)$ was determined according to the equation $[178 \mathrm{x} /(178 \mathrm{x}+$ $\left.M_{\text {pore }}\right)=$ mass loss]. The molecular weight of ANT is $178 \mathrm{~g} \mathrm{~mol}^{-1}$. The 'molecular weight' of the pore $\left(\mathrm{M}_{\text {pore }}\right)$ of MAF-6, ZIF-71, and MFM-
300 (Sc) are 3060, 4044, and $1800 \mathrm{~g} \mathrm{~mol}^{-1}$, respectively, calculated from the crystallographic data (CCDC code: MECWOH, GITVIP, and EXEQAA, respectively). $\mathrm{N}_{2}$ physisorption isotherms were measured at $77 \mathrm{~K}$ using a Micromeritics 3 Flex instrument. The samples were degassed before measurements at $150^{\circ} \mathrm{C}$ under a dynamic vacuum $\left(10^{-2} \mathrm{mbar}\right)$ for $12 \mathrm{~h}$. The ANT, 2-MA, or 9-MA-loaded samples were degassed at room temperature under dynamic vacuum $\left(10^{-2} \mathrm{mbar}\right)$ for $12 \mathrm{~h}$. The BET method for all the materials except SBA was applied in the region between 0.005 and $0.05 \mathrm{P} / \mathrm{P}_{0}$, consistent with the consistency criteria for microporous materials. ${ }^{[39]}$ The BET method for SBA and ANT@SBA was applied in the region between 0.1 and $0.3 \mathrm{P} / \mathrm{P}_{0}$. Fluorescence emission spectra were recorded on an FLS 980 fluorescence spectrophotometer (Edinburgh Instruments, Photonics division). The kinetics of the changes in fluorescence emission was recorded in situ by monitoring the emission intensity changes upon the excitation light irradiation $(\lambda=$ $360 \mathrm{~nm})$.

\section{Acknowledgements}

M.T. is grateful for the financial support from a Marie SkłodowskaCurie Individual Fellowship (No. 708439, VAPOMOF). R.A. acknowledges funding from the European Research Council (No. 716472, VAPORE) and the Research Foundation Flanders (FWO) for funding in the research projects G083016N and $1501618 \mathrm{~N}$, and the infrastructure project GOH0716N. KU Leuven is acknowledged for funding in the project C32/18/056 and C14/20/085. J.H. gratefully acknowledges the financial support of the Flemish Government through long-term structural funding Methusalem ((CASAS2, Meth/15/04) as well as the financial support from the MPI as MPI fellow.

\section{Conflict of Interest}

The authors declare no competing financial interests.

Keywords: Anthracene - Host-guest system - Metal-organic frameworks . Photoswitching

[1] M. Sauer, PNAS 2005, 102, 9433-9434.

[2] M.-M. Russew, S. Hecht, Adv. Mater. 2010, 22, 3348-3360.

[3] S. van de Linde, M. Sauer, Chem. Soc. Rev. 2014, 43, 1076-1087.

[4] C. Zhang, M.-H. Du, H.-P. Cheng, X.-G. Zhang, A. E. Roitberg, J. L. Krause, Phys. Rev. Lett. 2004, 92, 158301.

[5] M. Irie, T. Fukaminato, K. Matsuda, S. Kobatake, Chem. Rev. 2014, 114, 12174-12277.

[6] H. Bouas-Laurent, A. Castellan, J.-P. Desvergne, R. Lapouyade, Chem. Soc. Rev. 2000, 29, 43-55.

[7] H. Bouas-Laurent, A. Castellan, J.-P. Desvergne, R. Lapouyade, Chem. Soc. Rev. 2001, 30, 248-263.

[8] M. Irie, T. Fukaminato, T. Sasaki, N. Tamai, T. Kawai, Nature 2002, 420, 759-760.

[9] T. Fukaminato, T. Sasaki, T. Kawai, N. Tamai, M. Irie, J. Am. Chem. Soc. 2004, 126, 14843-14849.

[10] M. Heilemann, P. Dedecker, J. Hofkens, M. Sauer, Laser Photonics Rev. 2009, 3, 180-202.

[11] T. Fukaminato, J. Photochem. Photobiol. C 2011, 12, 177-208.

[12] R. Haldar, L. Heinke, C. Wöll, Adv. Mater. 2020, 32, 1905227.

[13] A. S. Kumar, T. Ye, T. Takami, B.-C. Yu, A. K. Flatt, J. M. Tour, P. S. Weiss, Nano Lett. 2008, 8, 1644-1648. 
[14] H. A. Schwartz, U. Ruschewitz, L. Heinke, Photochem. Photobiol. Sci. 2018, 17, 864-873.

[15] B. Gui, Y. Meng, Y. Xie, K. Du, A. C.-H. Sue, C. Wang, Macromol. Rapid Commun. 2018, 39, 1700388.

[16] A. M. Rice, C. R. Martin, V. A. Galitskiy, A. A. Berseneva, G. A. Leith, N. B. Shustova, Chem. Rev. 2020, 120, 8790-8813.

[17] G. Tabacchi, ChemPhysChem 2018, 19, 1249-1297.

[18] K. Weh, M. Noack, K. Hoffmann, K.-P. Schröder, J. Caro, Microporous Mesoporous Mater. 2002, 54, 15-26.

[19] S. Garg, H. Schwartz, M. Kozlowska, A. B. Kanj, K. Müller, W. Wenzel, U. Ruschewitz, L. Heinke, Angew. Chem. Int. Ed. 2019, 58, 1193-1197; Angew. Chem. 2019, 131, 1205-1210.

[20] D. Hermann, H. A. Schwartz, M. Werker, D. Schaniel, U. Ruschewitz, Chem. Eur. J. 2019, 25, 3606-3616.

[21] Z. Li, G. Wang, Y. Ye, B. Li, H. Li, B. Chen, Angew. Chem. Int. Ed. 2019, 131, 18193-18199.

[22] Z. Shao, Q. Wu, X. Han, Y. Zhao, Q. Xie, H. Wang, H. Hou, Chem. Commun. 2019, 55, 10948-10951.

[23] S. R. Batten, N. R. Champness, X.-M. Chen, J. Garcia-Martinez, S. Kitagawa, L. Öhrström, M. O'Keeffe, M. Paik Suh, J. Reedijk, Pure Appl. Chem. 2013, 85, 1715-1724.

[24] H. Furukawa, K. E. Cordova, M. O'Keeffe, O. M. Yaghi, Science 2013, 341 1230444.

[25] M. Tu, H. Reinsch, S. Rodríguez-Hermida, R. Verbeke, T. Stassin, W. Egger, M. Dickmann, B. Dieu, J. Hofkens, I. F. J. Vankelecom, N. Stock, R. Ameloot, Angew. Chem. Int. Ed. 2019, 58, 2423-2427; Angew. Chem. 2019, 131, 2445-2449.

[26] X.-C. Huang, Y.-Y. Lin, J.-P. Zhang, X.-M. Chen, Angew. Chem. Int. Ed. 2006, 45, 1557-1559; Angew. Chem. 2006, 118, 1587-1589.

[27] R. Banerjee, A. Phan, B. Wang, C. Knobler, H. Furukawa, M. O'Keeffe, O. M. Yaghi, Science 2008, 319, 939-943.

[28] C.-T. He, L. Jiang, Z.-M. Ye, R. Krishna, Z.-S. Zhong, P.-Q. Liao, J. Xu, G. Ouyang, J.-P. Zhang, X.-M. Chen, J. Am. Chem. Soc. 2015, 137, 72177223.

[29] T. Hinoue, Y. Shigenoi, M. Sugino, Y. Mizobe, I. Hisaki, M. Miyata, N. Tohnai, Chem. Eur. J. 2012, 18, 4634-4643.
[30] M. Sugino, Y. Araki, K. Hatanaka, I. Hisaki, M. Miyata, N. Tohnai, Cryst. Growth Des. 2013, 13, 4986-4992.

[31] F. Fennel, J. Gershberg, M. Stolte, F. Würthner, Phys. Chem. Chem. Phys. 2018, 20, 7612-7620.

[32] I. A. Ibarra, S. Yang, X. Lin, A. J. Blake, P. J. Rizkallah, H. Nowell, D. R. Allan, N. R. Champness, P. Hubberstey, M. Schröder, Chem. Commun. 2011, 47, 8304-8306.

[33] F. Márquez, H. García, E. Palomares, L. Fernández, A. Corma, J. Am. Chem. Soc. 2000, 122, 6520-6521.

[34] D. Zhao, J. Feng, Q. Huo, N. Melosh, G. H. Fredrickson, B. F. Chmelka, G. D. Stucky, Science 1998, 279, 548-552.

[35] J. M. Robertson, W. H. Bragg, Proc. R. Soc. London Ser. A 1933, 140, 7998.

[36] L. Yin, D. Wu, Z. Yao, S. Feng, M. Zhang, Y. Gao, Thermochim. Acta 2013, $565,72-81$.

[37] B. Stevens, Spectrochim. Acta 1962, 18, 439-448.

[38] I. Stassen, M. Styles, G. Grenci, H. V. Gorp, W. Vanderlinden, S. D. Feyter P. Falcaro, D. D. Vos, P. Vereecken, R. Ameloot, Nat. Mater. 2016, 15, 304-310.

[39] T. Stassin, I. Stassen, J. Marreiros, A. J. Cruz, R. Verbeke, M. Tu, H. Reinsch, M. Dickmann, W. Egger, I. F. J. Vankelecom, D. E. De Vos, R. Ameloot, Chem. Mater. 2020, 32, 1784-1793.

[40] R. P. Lively, M. E. Dose, J. A. Thompson, B. A. McCool, R. R. Chance, W. J. Koros, Chem. Commun. 2011, 47, 8667-8669.

[41] J. Cravillon, S. Münzer, S.-J. Lohmeier, A. Feldhoff, K. Huber, M. Wiebcke, Chem. Mater. 2009, 21, 1410-1412.

[42] R. Locus, D. Verboekend, M. d'Halluin, M. Dusselier, Y. Liao, N. Nuttens, T. Jaumann, S. Oswald, L. Mafra, L. Giebeler, B. Sels, ChemCatChem 2018, 10, 1385-1397.

[43] K. S. Walton, R. Q. Snurr, J. Am. Chem. Soc. 2007, 129, 8552-8556. 